To the Editors:

\title{
Scientific research-based evidence used in drug promotion material distributed in Sri Lanka
}

\author{
M Kommalage', D Nayanarasie', S Basnayake ${ }^{1}$
}

Ceylon Medical Journal 2016; 61: 199

http://doi.org/10.4038/cmj.v61i4.8394

Drug promotion materials (DPM) are commonly used by pharmaceutical companies to provide information to doctors about drugs. The information in DPM may have an impact on treatment even more than the biomedical quality of the drug [1].

In the drug promotion process, pharmaceutical companies make many claims about the efficacy, safety and 'quality' of the products. These claims should be clear, valid and justifiable. In scientific literature, each claim should be supported by evidence. Therefore, they are expected to provide evidence to support the claims made in DPM. The high quality references increase the authenti-city of the evidence. The aim of this study was to examine the quality of scientific supportive evidence used in DPM in Sri Lanka.

A group of physicians, paediatricians, and general practitioners were informed about the study through emails and requested to collect DPM. DPM received from these doctors were included in the study. Information presented in those DPM was extracted by two researchers working together. Another researcher checked and verified the extracted information.

We studied 211 DPM collected from August to October 2015. Two hundred and eight DPM out of 211 had one or more claims about 'quality' of the product. From 208 DPM which claimed about 'quality' of the product, only $167(80.3 \%)$ provided evidence, as references, for the claim.

A total of 569 references were found in those 167 DPM. Five hundred and one were journal articles (88.0\%). 497 were PubMed indexed (87.3\%) journals and 453 (79.6\%) were ISI indexed journals. This ISI indexed journals have an Impact Factor and a higher impact factor indicates a higher ranked journal. Ninety three journals $(18 \%)$ had an impact factor of more than 10,160 journals (32\%) had an impact factor of more than 5 while 341

journals $(68 \%)$ had less than five or no impact factor. Considering the year of publication of the journal articles, $91.5 \%$ articles were published in 2010 or before and $44.2 \%$ were published during 2000 or before.

Twenty references $(3.5 \%)$ could not be traced using the given information in DPM. Twenty one were non-journal websites, 16 of these webpages $(76 \%)$ were not available, directed to another database or were altered in such a way that it did not provide the expected information. The text size used for the reference list was divided into four categories. Compared to the citation text, $2.4 \%$ had same size text in reference list, $20.4 \%$ had half or more size list, $75.4 \%$ had less than half size text and $1.8 \%$ could not be read without a magnifying glass (or somebody with very good vision). The name and address of manu-facturer or distributor was mentioned in only 185 of DPM $(87.6 \%)$.

Some DPM claims about 'quality' of the drug was not supported by evidence. Considerable number of DPM used poor quality scientific research as references. Some of these references could not be traced. This study empha-sises the importance of searching related scientific literature by user themselves before accepting claims in DPM.

\section{Conflicts of interest}

There are no conflicts of interest.

\section{Reference}

1. Prosser HS,. Almond, Walley T. Influences on GPs' decision to prescribe new drugs - the importance of who says what. Fam Pract 2003; 20: 61-8.

${ }^{1}$ Department of Physiology, Faculty of Medicine, University of Ruhuna, Galle, Sri Lanka.

Correspondence: MK, e-mail: <mahinda1@gmail.com>. Received 12 May 2016 and revised version accepted 25 August 2016.

This is an open-access article distributed under the terms of the Creative Commons Attribution License, which permits unrestricted use, distribution, and reproduction in any medium, provided the original author and source are credited. 